\title{
COMPLICACIONES DE LAS UVEÍTIS: PREVALENCIA Y FACTORES DE RIESGO EN UNA SERIE DE 398 CASOS
}

\author{
COMPLICATIONS OF UVEITIS: PREVALENCE AND RISK \\ FACTORS IN A SERIE OF 398 CASES
}

\author{
PRIETO-DEL-CURA M ${ }^{1}$, GONZÁLEZ-GUIJARRO J²
}

\begin{abstract}
RESUMEN
Objetivo: Estimar el porcentaje de complicaciones y establecer factores de riesgo para padecerlas en los pacientes con uveítis.

Método: Estudio prospectivo de cohortes de 398 pacientes con seguimiento superior a 1 mes desde Enero del 2000 hasta Octubre del 2007. Se evaluaron los datos demográficos, lateralidad, curso (agudo, subagudo crónico), localización, diagnóstico (idiopática, infecciosa, de base ocular, de base sistémica, otras) y el desarrollo de complicaciones (catarata, edema macular quístico, complicaciones maculares, complicaciones retinianas y otras).

Resultados: Tras un seguimiento medio de 35,2 meses (1-160), el 25\% desarrollaron una o más de las siguientes complicaciones: catarata $8,5 \%$, edema macular $6,1 \%$, complicaciones maculares (membrana epirretiniana, membrana neovascular coroidea, necrosis macular) 4,1\%, complicaciones retinianas (desprendimiento de retina, neovasos retinianos, hemovítreo, desgarros retinianos, oclusiones vasculares retinianas) $4,1 \%$, glaucoma o hipertensión ocular 3,6\% y otras 1,5\%. Los factores de riesgo para desarrollarlas fueron el curso crónico OR 6,37 (3,25-12,47) $\mathrm{P}<0,0001$, la bilateralidad OR $1,98(1,03-3,08) \mathrm{P}=0,039$, el curso agudo recidivante $\mathrm{OR} 1,94(0,02-4,08) \mathrm{P}=0,08$ y la panuveítis OR 1,92 $(0,99-4,80) \mathrm{P}=0,161$.
\end{abstract}

\footnotetext{
Recibido: 31/5/08. Aceptado: 28/10/09.

Hospital de La Princesa. Servicio de Oftalmología. Madrid. España.

1 Licenciada en Medicina.

2 Doctor en Medicina.

Correspondencia:

M. Prieto del Cura

Hospital de La Princesa. Servicio de Oftalmología

C/. Diego de León, 62

28006 Madrid

España

E-mail: marprieto20@yahoo.com
}

\begin{abstract}
Objective: To estimate the rate of complications and establish risk factors for their development in patients with uveitis.

Methods: Prospective study of a cohort of 398 patients (413 eyes) that were reviewed from January 2000 to October 2007 and monitored during a period of at least one month. Demographic data, laterality, course (acute, subacute, chronic), location, diagnosis (idiopathic, infectious, ocular origin, systemic origin, others) and development of complications (cataract, cystoid macular edema, macular complications, retinal complications and others) were evaluated.

Results: After a mean follow-up of 35.2 months (range 1-160), $25 \%$ of patients developed one or more of the following complications: cataract $8.5 \%$, macular edema $6.1 \%$, macular complications (epiretinal membranes, choroidal neovascular membranes, macular necrosis) $4.1 \%$, retinal complications (retinal detachment, retinal neovascularization, vitreous hemorrhage, retinal tears, retinal vascular occlusions) $4.1 \%$, glaucoma or ocular hypertension $3.6 \%$, and others $1.5 \%$. Risk factors for development of uveitis complications were chronic course OR 6.37 (3.25-12.47) P $<0.0001$, bilaterality OR $1.98(1.03-3.08) \mathrm{P}=0.039$, subacute course OR 1.94 (0.02-4.08) $\mathrm{P}=0.08$, and panuveitis OR 1,92 (0.99-4.80) $\mathrm{P}=0.161$.
\end{abstract}


Conclusiones: En nuestros pacientes las uveítis crónicas y las bilaterales son las que más riesgo tienen de sufrir complicaciones de las que la catarata es la más frecuente.

Palabras clave: Uveítis, catarata, factores de riesgo, complicaciones.
Conclusions: In our patients, cases involving chronic and bilateral uveitis were more prone to develop complications, with cataracts being the most frequent complication (Arch Soc Esp Oftalmol 2009; 84: 523-528).

Key words: Uveitis, cataract, risk factors, complications.

\section{INTRODUCCIÓN}

La incidencia de uveítis, en los países desarrollados, es de 45 casos por 100.000 habitantes y año, con una prevalencia de 70 por $100.000(1,2)$, siendo la causa del $10-15 \%$ de los nuevos casos de ceguera en USA.

Las complicaciones que ocasionan son la causa de ceguera y alteraciones graves de la función visual en estos pacientes, destacando entre éstas el edema macular quístico irreversible, la catarata, el glaucoma, las lesiones de la mácula y del nervio óptico, distintas anomalías vasculares retinianas, o el desprendimiento de retina (3).

Con frecuencia las complicaciones están presentes al momento del diagnóstico, pero cuando no es así el grado y características de la inflamación ocular en el examen inicial puede ser un importante factor pronóstico para el desarrollo de complicaciones oculares y en consecuencia de pérdida de agudeza visual durante el seguimiento.

El objetivo de nuestro estudio es estimar el porcentaje de complicaciones y establecer factores de riesgo para padecerlas en una serie de pacientes con uveítis.

\section{SUJETOS, MATERIAL Y MÉTODOS}

Hemos efectuado un estudio prospectivo de cohortes descriptivo en 398 pacientes, diagnosticados de uveítis en la sección de úvea del Servicio de Oftalmología del Hospital Universitario de la Princesa, mayores de 14 años con seguimiento superior a un mes desde enero de 2000 hasta octubre de 2007.

Los datos se obtuvieron de una base de datos informatizada. Como variables demográficas se recogieron la edad en años y el sexo. Las variables oftalmológicas recogidas fueron:

1. Localización primaria: siguiendo los criterios de clasificación anatómica del The Standardization of Uveitis Nomenclature (SUN) Working Group ${ }^{(4)}$ que son: uveítis anterior (iritis, iridociclitis), uveítis intermedia (pars planitis, ciclitis posterior, hialitis) uveítis posterior y panuveítis.

2. Curso clínico: según los criterios del SUN working group (4) cada episodio se divide en: agudo, recurrente o subagudo y crónico.

3. Lateralidad: unilateral o bilateral.

4. Diagnóstico: con el perfil clínico del paciente detallado se genera un listado de posibilidades diagnósticas que fueron agrupadas en:

- Idiopática.

- Infecciosa.

- De base ocular: con la intención de aunar criterios incluimos: crisis glaucomatociclíticas, pars planitis, ciclitis heterocrómica de Fuchs, asociadas al HLA B 27 sin manifestaciones sitémicas, los síndromes de puntos blancos, coroidopatía en perdigonada, enfermedad de Eales, postquirúrgicas no infecciosas y la oftalmía simpática.

- De base sistémica: cuando se acompaña o en el curso de una enfermedad sistémica autoinmune o de etiopátogenia inmunológica: síndrome de Bechet, espondiloartropatías seronegativas, asociadas a enfermedad inflamatoria ocular, síndrome de Sjogren, psoriasis, esclerosis múltiple, enfermedad de Harada, artritis crónica juvenil, artritis reumatoide, sarcoidosis y diabetes.

- Otras: incluimos los síndromes mascarada, farmacológicas, oclusiones de rama arterial recidivantes idiopáticas.

6. Complicaciones:

1) Catarata: definida como la presencia de 1+ esclerosis nuclear o $1+$ cambio cortical o subcapsular posterior.

2) Glaucoma o hipertensión ocular: definido como PIO mayor de $21 \mathrm{mmHg}$ o daño glaucomatoso en el nervio óptico que requiere tratamiento médico o quirúrgico antiglaucomatoso.

3) Edema macular: presencia de engrosamiento macular con o sin formaciones quísticas que puede 
Tabla I. Distribución de la edad

\begin{tabular}{lcccc}
\hline & Mediana (RIC) & En complicados & En no complicados & P (Mann-Whitney) \\
\hline Edad & $44(31-59)$ & $41,5(31-55)$ & $44(32-61)$ & 0,35 \\
\hline \hline
\end{tabular}

ser observado con exploración clínica, angiográfica y/o por OCT.

4) Maculares: membrana neovascular coroidea (MNVC), membrana epirretiniana (MER), necrosis macular.

5) Retinianas: desprendimiento de retina, desgarros retinianos, neovasos retinianos, oclusiones vasculares y hemovítreo.

6) Otras: donde se incluye la queratopatía en banda y las sinequias posteriores mayores de $180^{\circ}$.

Para determinar si existen diferencias entre los grupos de pacientes sin complicaciones y el grupo de pacientes con ellas, así como los factores de riesgo para padecerlas, analizamos las variables estudiadas. El análisis estadístico se realizó a través de las pruebas de $\chi^{2}$ para testear la asociación entre las variables cualitativas y el desarrollo de complicaciones y Mann-Whitney (tras test de kurtosis y asimetría) para la comparación de edad entre los pacientes con y sin complicaciones.

Como medida de efecto utilizamos la OR (odds ratio) con su correspondiente intervalo de confianza al $95 \%$. Con el objeto de conocer el efecto conjunto de las variables utilizamos la regresión logística, comprobando el ajuste del modelo con el test de Hosmer-Lemeshow.

En todos los casos consideramos como estadísticamente significativos los $\mathrm{p}$ valores $<0,05$.

Todos los análisis se realizaron con la ayuda del programa SPSS v15.0 (SPSS Inc Chicago Ill), Intercooled Stata 9.1 para Windows, y EpiDat 3.1.

\section{RESULTADOS}

Se analizaron 398 pacientes con uveítis, el 47\% de los episodios ocurrieron en varones, la mediana de edad fue 44 años (RIC 31-59), y el seguimiento mediano 22 meses (RIC 4-60).

Cien $(25,1 \%)$ de los pacientes analizados tuvieron una o más complicaciones. El 50,4\% ocurrieron en mujeres, la mediana de edad fue 41,5 años (RIC 31-55), con seguimiento mediano de 49 meses (RIC 19-72) (tabla I).

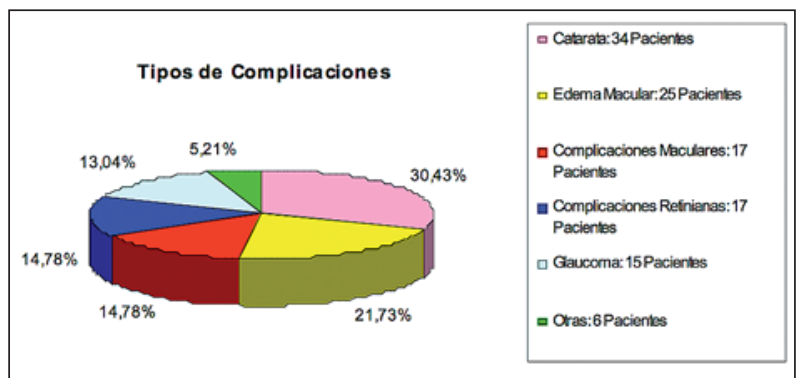

Gráfico 1: Prevalencia de las complicaciones.

Ninguna de las variables demográficas (edad y sexo) pudo ser considerada factor de riesgo para padecer complicaciones.

Para el estudio hemos analizado las complicaciones por lo que se han duplicado aquellos pacientes que tuvieron más de una. Las complicaciones estudiadas están recogidas en la tabla II y grafico1.

Tabla II. Prevalencia de las complicaciones

\begin{tabular}{lcc}
\hline Complicación & Pacientes & Porcentaje \\
\hline Catarata & 34 & $8,50 \%$ \\
\hline Edema macular & 25 & $6,10 \%$ \\
\hline Complicaciones Maculares & & \\
$\quad$ Total & 17 & $4,10 \%$ \\
Membrana neovascular coroidea & 8 & \\
Membrana epirretiniana & 5 & \\
Necrosis Macular & 4 & \\
\hline Complicaciones retinianas & & \\
Total & 17 & $4,10 \%$ \\
Desprendimiento de retina & 8 & \\
Desgarros retinianos & 1 & \\
Hemovitreo & 1 & \\
Neovasos retinianos & 2 & \\
Oclusiones Vasculares Retinianas & 5 & \\
\hline Glaucoma o Hipertensión ocular & 15 & $3,60 \%$ \\
\hline Otras & & \\
Total & 6 & $1,50 \%$ \\
Queratopatía en banda & 2 & \\
Sinequias posteriores mayores a 180 & 4 & \\
\hline \hline
\end{tabular}


Tabla III. Distribución de las variables y análisis de la asociación con complicaciones

\begin{tabular}{|c|c|c|c|c|c|c|c|}
\hline \multirow[b]{2}{*}{ Variable } & \multicolumn{5}{|c|}{ Análisis univariado } & \multicolumn{2}{|c|}{ Análisis multivariado } \\
\hline & Categoría & $\begin{array}{c}\% \\
\text { muestra }\end{array}$ & $\begin{array}{c}\% \\
\text { complicaciones }\end{array}$ & OR (IC95\%) & $P$ valor & OR (IC95\%) & $\mathbf{P}$ valor \\
\hline \multirow[t]{2}{*}{ Sexo } & Mujer & 53 & 26,5 & $0,87(0,56-1,32)$ & 0,512 & \multirow{2}{*}{\multicolumn{2}{|c|}{ No mejora el modelo }} \\
\hline & Varón & 47 & 29,4 & & & & \\
\hline \multirow[t]{2}{*}{ Lateralidad } & Unilateral & 62,7 & 18,5 & \multirow[t]{2}{*}{$3,53(2,26-5,62)$} & \multirow[t]{2}{*}{$<0,001$} & 1 & \\
\hline & Bilateral & 37,3 & 45,1 & & & $1,78(1,03-3,09)$ & 0,039 \\
\hline \multirow[t]{5}{*}{ Localización } & Anterior & 58,1 & 20,4 & 1 & & 1 & \\
\hline & Intermedia & 9,7 & 40,0 & $2,59(1,28-5,26)$ & 0,008 & $0,73(0,32-1,68)$ & 0,464 \\
\hline & Posterior & 24,5 & 37,6 & $2,35(1,41-3,91)$ & 0,001 & $1,58(0,83-3,03)$ & 0,164 \\
\hline & Panuveítis & 7,8 & 37,5 & $2,34(1,07-5,11)$ & 0,033 & $1,92(0,77-4,80)$ & 0,161 \\
\hline & Total & 100 & & & 0,001 & & \\
\hline \multirow[t]{4}{*}{ Curso clínico } & Agudo & 42 & 12,6 & 1 & & 1 & \\
\hline & Agudo recidivante & 24,9 & 20,2 & $1,76(0,91-3,42)$ & 0,096 & $1,94(0,92-4,08)$ & 0,080 \\
\hline & Crónico & 33,2 & 51,5 & $7,39(4,19-13,02)$ & $<0,001$ & $6,37(3,26-12,47)$ & $<0,001$ \\
\hline & Total & 100 & & 1 & $<0,001$ & & \\
\hline \multirow[t]{6}{*}{ Diagnóstico } & Idiopática & 26,9 & 25,5 & 1 & & \multirow{6}{*}{\multicolumn{2}{|c|}{ No mejora el modelo }} \\
\hline & Infecciosa & 28,1 & 24,3 & $0,94(0,52-1,72)$ & 0,848 & & \\
\hline & Autoinmune ocular & 13,7 & 39,3 & $1,89(0,96-3,75)$ & 0,066 & & \\
\hline & Autoinmune sistémica & 25,9 & 30,2 & $1,27(0,70-2,29)$ & 0,437 & & \\
\hline & Otras & 5,4 & 22,7 & $0,86(0,30-2,47)$ & 0,787 & & \\
\hline & Total & 100 & & & 0,268 & & \\
\hline
\end{tabular}

De las variables estudiadas, la cronicidad (OR 6,37 [IC95\% 3,26-12,47\%] P < 0,001) y la bilateralidad (OR 1,78 [IC95\% 1,03-3,09] $\mathrm{P}=0,039)$ son factores de riesgo estadísticamente significativos para desarrollar complicaciones en nuestros pacientes.

Existe una tendencia en las uveítis de curso recidivante (OR 1,94 [IC95\% 0,92-4,08] P=0,08) sin llegar a ser estadísticamente significativa.

En el análisis de la localización de la inflamación, la localización posterior es la que mayores complicaciones asocia, seguida por la panuveítis que tiene un alto valor de asociación con las complicaciones $\sin$ ser estadísticamente significativa (OR 1,92 [0,77-4,80] $\mathrm{P}=0,161$ ) (tabla III).

$\mathrm{Al}$ analizar si existía alguna asociación estadística entre una determinada complicación con las variables demográficas y oftalmológicas estudiadas, ninguna fue significativa.

\section{DISCUSIÓN}

Las complicaciones de las uveítis son una causa de importante morbilidad ocular. Rothova (1) en 1996 en una serie de 582 casos observó que el $35 \%$ de los pacientes con uveítis tenían pérdida visual significativa y el $4 \%$ ceguera legal, donde las complicaciones aparecen en más del $50 \%$ (1). En nuestro estudio, con un seguimiento medio de 4 años, similar al de Rothova, las complicaciones aparecieron en el $25 \%$ de los pacientes, esta diferencia se puede deber a que en una década el mejor conocimiento de la historia natural de las uveítis y la eficacia de los nuevos tratamientos inmunosupresores han hecho disminuir el porcentaje de las mismas. Otra razón que explica esta diferencia entre ambos estudios es que nuestro hospital no es centro de referencia para pacientes con uveítis y probablemente manejamos mayor porcentaje de episodios agudos, por tanto las características de los pacientes pueden no ser comparables. Nuestro porcentaje es algo mayor (20\%) al observarlo en una serie japonesa realizada en el año 2003, con un seguimiento inferior ( 2 años) y con mayor porcentaje de uveítis posteriores (5).

Los pacientes entre 25 y 44 años son el grupo de edad con mayor incidencia (1), aunque la mediana de edad de nuestro estudio es de 44 años al llevarse a acabo en un hospital donde habitualmente no se atiende a población infantil. 
Cuando la inflamación no revierte con el tratamiento, o cursa con episodios recidivantes, el riesgo de aparición de complicaciones se incrementa y en esta línea están los resultados de nuestro estudio. Así el riesgo de tener complicaciones es mayor cuando la uveítis se cronifica o es bilateral y además existe una importante asociación sin llegar a ser estadísticamente significativa en aquellas con un curso clínico agudo recidivante.

Se ha descrito que los pacientes con panuveítis son los que más complicaciones asocian y peor pronóstico visual tienen $(1,6)$, y así ocurre en nuestra serie con una fuerte asociación estadística con la presencia de complicaciones, por lo que en estos pacientes debemos establecer una estrategia de tratamiento agresiva para intentar evitarlas.

Entre las complicaciones desarrolladas en nuestro estudio fue la catarata la más frecuente con un $30 \%$ del total de complicaciones, seguida por el edema macular con un 21,3\% y de la hipertensión ocular/glaucoma con un 13\%. Distribución no muy diferente a la descrita previamente (1) aunque con mayor porcentaje de cataratas (frente al 19\%) y menos de edema macular (frente al 26\%). En otras series el edema macular quístico supone la principal causa de disminución de la agudeza visual central en las uveítis intermedias y la complicación más frecuente $(21,7 \%)$ seguida de las cataratas $(13,0 \%)$, que pueden ser secundarias tanto al tratamiento con corticoides como a la propia enfermedad inflamatoria (6). Esta diferente distribución entre series puede tener los mismos condicionantes mencionados previamente.

Se admite que, tanto la inflamación inicial como la persistencia de la misma y por tanto las complicaciones asociadas, son el resultado de una compleja combinación de factores genéticos, ambientales e inmunológicos (3). Con este estudio se ha pretendi- do arrojar más luz sobre esa compleja combinación de factores causantes o asociados a la posibilidad de desarrollar complicaciones en el curso de una uveítis ya que existen pocas publicaciones al respecto, en particular en nuestro medio.

En conclusión, en nuestra experiencia las uveítis crónicas y bilaterales son las que mayor riesgo tienen de sufrir complicaciones y de ellas, la catarata ha sido la complicación más frecuente. Las demás variables estudiadas no se han podido considerar factores de riesgo estadísticamente significativos a pesar de que algunas categorías presentan tendencias interesantes. Probablemente esto guarda relación con el pequeño tamaño muestral de algunos grupos, que hace difícil encontrar diferencias estadísticamente significativas en caso de asociaciones moderadas.

\section{BIBLIOGRAFÍA}

1. Rothova A, Suttorp-van Schulten MS, Frits Tretters W, Kijlstra A. Causes and frequency of blindness in patients with intraocular inflammatory disease. $\mathrm{Br} J$ Ophthalmol 1996; 80: 332-336.

2. Foster SC, Vitale AT. Diagnosis and treatment of uveitis. 1st ed. USA: W.B. Saunders company; 2002.

3. Gegúndez Fernández. JA. Ancillary tests in uveitis. Arch Soc Esp Oftalmol 2003; 78: 643-646.

4. Jabs DA, Nussenblatt RB, Rosenbaum JT; Standardization of uveitis nomenclatura (SUN) Working Group. Standardization of uveitis nomenclature for reporting clinical data. Results of the First International Workshop. Am J Ophthalmol 2005;140: 509-516.

5. Wakabayashi T, Morimura Y, Miyamoto Y, Okada AA. Changing patterns of intraocular inflammatory disease in Japan. Ocul Immunol Inflamm 2003; 11: 277-286.

6. Frau Rotger E, Corretger Ruhi X, Mateos Poch JM, Olea Vallejo JL. Intermediate uveitis. Retrospective study. Arch Soc Esp Oftalmol 1999; 74: 519-523. 